\title{
Construction of a virulent, green fluorescent protein- tagged Yersinia ruckeri and detection in trout tissues after intraperitoneal and immersion challenge
}

\author{
Timothy J. Welch*, Gregory D. Wiens \\ National Center for Cool and Cold Water Aquaculture, Agricultural Research Service, US Department of Agriculture, \\ 11861 Leetown Road, Kearneysville, West Virginia 25430, USA
}

\begin{abstract}
A green fluorescent protein (GFP) expressing strain of Yersinia ruckeri was created by the transposition of a Tn10-GFP-kan cassette into the genome of Y. ruckeri Strain YRNC10. The derivative, YRNC10-gfp, was highly GFP fluorescent, retained the $g f p-k m$ marker in the absence of kanamycin selection, and exhibited in vitro growth kinetics similar to those of the wild type strain. YRNC10-gfp colonized and caused mortality in immersion and intraperitoneally challenged rainbow trout Oncorhynchus mykiss, although it was modestly attenuated compared to the wild type strain. The distribution and location of YRNC10-gfp in infected fish was visualized by epifluorescence microscopy. Abundant extracellular bacteria and a small number of intracellular bacteria were observed in the kidney, spleen and peripheral blood. To determine the percentage of trout cells containing intracellular bacteria, GFP fluorescence was measured by flow cytometry. A small population of GFP positive leukocytes was detected in peripheral blood $(1.6 \%)$, spleen $(1.1 \%)$ and anterior kidney $(0.4 \%)$ tissues. In summary, this is the first report of the construction of a virulent, GFP-tagged Y. ruckeri, which may be a useful model for detecting and imaging the interactions between an aquatic pathogen and the natural salmonid host.
\end{abstract}

KEY WORDS: Yersinia ruckeri · Green fluorescent protein · Flow cytometry · Epifluorescence microscopy

Resale or republication not permitted without written consent of the publisher

\section{INTRODUCTION}

Yersinia ruckeri was first recognized as a pathogen of rainbow trout in the Northwestern United States in the 1950s and has since appeared throughout North America and Europe, primarily in areas where trout are intensively cultured (Stevenson 1997). This Gramnegative bacterium is the causative agent of Enteric Redmouth Disease (ERM), an acute hemorrhagic septicemia. ERM outbreaks are often associated with poor water quality, excessive stocking densities, handling of fish and the occurrence of environmental stressors. Y. ruckeri can also persist in an asymptomatic carrier state in which bacteria are shed in the faeces, with carrier fish serving as a reservoir for recurrent infections, often occurring at times of stress (Busch 1978, Stevenson 1997). Despite the early recognition of $Y$. ruckeri as an economically important pathogen of cultured salmonids, little work has been done to characterize the virulence mechanisms of this pathogen and as a result, a model for its pathogenesis has not yet been developed.

Genetic tagging of pathogenic microorganisms with the gene encoding the green fluorescent protein (GFP) from Aequorea victoria has become an important tool for elucidating the interactions between bacterial pathogens and their hosts (Valdivia \& Falkow 1996, Valdivia et al. 1996, Southward \& Surette 2002). GFP- 
tagging allows in situ detection of bacteria-host interactions in real time. GFP fluorescence associated with tagged cells can easily be visualized and quantified by epifluorescence microscopy, flow cytometry, spectrofluorometry or fluorescence colony counting (Chalfie et al. 1994, Valdivia \& Falkow 1996, Valdivia et al. 1996). More recently, artificial plasmids carrying the gene encoding the green fluorescent protein have been used to tag several fish pathogens (Ling et al. 2000, 2001, O'Toole et al. 2004), while these tagged strains have been useful for determining the hostpathogen interactions during the early stages of infection, the inherent instability of plasmid-mediated tags may limit their use in studies involving the long-term persistence of pathogens. Herein, we describe the use of a specialized transposon to construct a highly stable $g f p$-tagged derivative of Yersinia ruckeri and the use of this strain to visualize and quantify the interaction of this pathogen with trout immune cells.

\section{MATERIALS AND METHODS}

Bacterial strains and culture conditions. Yersinia ruckeri Strain YRNC10 is a Serotype 1 strain that was isolated from a moribund rainbow trout collected at a fish farm in North Carolina. YRNC10 and its derivatives were cultured at $24^{\circ} \mathrm{C}$ in trypticase soy broth (TSB), or trypticase soy agar (TSA). Escherichia coli strains were grown at $37^{\circ} \mathrm{C}$ in Luria broth or Luria agar. When required, antibiotics (Sigma) were added at the following concentrations: ampicillin $\left(100 \mu \mathrm{g} \mathrm{ml}^{-1}\right)$; kanamycin $\left(100 \mu \mathrm{g} \mathrm{ml}^{-1}\right.$ for Y. ruckeri and $50 \mu \mathrm{g} \mathrm{ml}^{-1}$ for E. coli).

Experimental fish. Rainbow trout were obtained from a commercial supplier (Trout Lodge, Washington). Stock and test fish were maintained at $16^{\circ} \mathrm{C}$ in freshwater under flow-through conditions and a fed a standard diet $5 \mathrm{~d}$ per week. Fish were anaesthetized with MS-222 (200 $\mathrm{mg} \mathrm{l}^{-1}$ ) and, when necessary, anaesthetized fish were euthanized by cervical dislocation.

Transposition of Tn10-gfp-kan cassette. Transposition was carried out using the mini-Tn10-gfp-kan transposon (Stretton et al. 1998), according to the method of de Lorenzo et al. (1990), with the following modifications. Conjugation mixes were incubated at $24^{\circ} \mathrm{C}$ for 12 to $18 \mathrm{~h}$ on TSA media, after which the cells were resuspended in TSB, diluted, and plated on TSA containing $100 \mu \mathrm{g} \mathrm{ml}^{-1}$ kanamycin to select for the transposon. Counter selection for the donor Escherichia coli strain was accomplished by incubation at $14^{\circ} \mathrm{C}$.

Immersion infection and estimation of bacterial loads in exposed fish. Rainbow trout were challenged by immersion exposure with approximately $10^{8}$ viable bacteria $\mathrm{ml}^{-1}$ tank water for $1 \mathrm{~h}$ in a static bath. Dissolved oxygen levels were maintained throughout the exposure procedure. Test fish were then maintained at $16^{\circ} \mathrm{C}$ in fresh water under flow-through conditions for $1 \mathrm{mo}$, and dead fish were removed and recorded daily. Necropsies were performed on each fish and mortality due to infection by Yersinia ruckeri was confirmed by microbiological analysis of spleen tissue. Bacterial loads in the spleens of exposed fish were quantified by direct plate count assay as follows. Whole organs were removed aseptically, weighed and homogenized in phosphate-buffered saline (PBS, $10 \mu \mathrm{mg}^{-1}$ ) by repeated passage through a $0.5 \mathrm{ml}$ syringe. Homogenates were then serially diluted and $100 \mathrm{ml}$ from each dilution plated on TSA. Colonies were enumerated after $24 \mathrm{~h}$ of incubation at $24^{\circ} \mathrm{C}$. Based on the amount of tissue homogenate plated, the detection limit of this assay was 1 bacterium per $10 \mathrm{mg}$ spleen tissue.

Statistical analysis. Bacterial load data was analyzed by 1-way ANOVA, and paired comparisons between wild-type (WT)- and GFP-infected fish were analyzed by Tukey's test. All statistical analysis was carried out using the GraphPad Prism 4 software package (GraphPad Software).

Cell samples and flow cytometry. For flow cytometry experiments, fish were intraperitoneally injected with $10^{6}$ bacteria in $0.1 \mathrm{ml}$ PBS. Tissues and blood were collected at $48 \mathrm{~h}$ post infection. Fish were anaesthetized with MS-222 (200 $\mathrm{mg} \mathrm{l}^{-1}$ ) and peripheral blood was collected from the caudal vein using a 20 gauge needle and a heparinized blood collection tube (BD Vacutainer). After blood removal, fish were euthanized by cervical dislocation and spleen and anterior kidney tissues removed aseptically. The samples were placed in $0.5 \mathrm{ml}$ of L15 medium (Sigma) containing $2 \%$ fetal bovine serum, FBS (Sigma) and homogenized using a $0.5 \mathrm{ml}$ syringe. Large tissue fragments were allowed to settle by incubation for $10 \mathrm{~min}_{\text {; }}$ the remaining suspended cells were transferred to a new tube and pelleted by centrifugation at $500 \times g$ for $10 \mathrm{~min}$ at $4^{\circ} \mathrm{C}$. Peripheral blood $(0.5 \mathrm{ml})$ was diluted 10-fold in L15$2 \%$ FBS prior to centrifugation. Pelleted cells were washed and resuspended in L15-2\% FBS medium containing $0.1 \%$ sodium azide. Erythrocytes were removed by discontinuous gradient centrifugation; $5 \mathrm{ml}$ of cell suspension was layered on $6 \mathrm{ml}$ histopaque 1077 (Sigma) in a $15 \mathrm{ml}$ tube and centrifuged at $500 \times g$ for $40 \mathrm{~min}$ at $4^{\circ} \mathrm{C}$. After centrifugation, white blood cells located at the interface were collected and washed twice with $10 \mathrm{ml}$ Dulbeccos PBS containing $2 \%$ FBS and $0.1 \%$ sodium azide. The light scattering and fluorescent properties of the cells were analyzed using a 3-color FACSCalibur (Becton Dickinson Biosciences). Data were analyzed using CellQuest Pro (Version 5.1.1). We recorded 20000 events for each sample, and gating was used to remove autofluorescent cells (FL1 and FL2 double positive) from the analysis. 


\section{RESULTS AND DISCUSSION}

\section{Generation of GFP tagged Yersinia ruckeri strain}

Transposition was used to deliver the mini Tn10-gfpkan cassette to the genome of Yersinia ruckeri, yielding a bank of approximately 6000 kanamycin resistant clones, each presumably containing a random mini Tn10-gfp-kan insertion. This transposon contains a promoterless $g f p$ gene and therefore $g f p$ expression occurs only if transposition results in transcriptional fusion to an active promoter within the recipient genome (Stretton et al. 1998). Of the 6000 transconjugants, 50 $(0.8 \%)$ were strongly GFP fluorescent under blue lightemitting diode (LED) light, indicating fusion to a strong $Y$. ruckeri promoter. Tn10-gfp-kan insertion in some transconjugants could compromise the ability of the resulting strain to colonize or cause disease; this may occur if the transposon insertion has altered the expression of a gene involved in either of these processes. Therefore, an in vivo selection strategy was used to enrich a tagged clone that had retained its ability to colonize rainbow trout. For this, the 50 GFP positive clones were grown individually to stationary phase, pooled, and the resulting mixture used to challenge rainbow trout by immersion. Moribund fish were observed $6 \mathrm{~d}$ after exposure, and fluorescence microscopy revealed high levels of GFP positive bacteria in the spleen and anterior kidney of these individuals. Standard microbiological methods were used to isolate bacteria from these tissues into pure culture, and a single strongly GFP-fluorescent clone (YRNC10-gfp) was selected for further study. YRNC10-gfp displayed strong GFP fluorescence in all phases of growth in liquid as well as on solid culture media, and had no discernible abnormalities in growth on these media (data not shown).

\section{YRNC10-gfp retention of pathogenicity for rainbow trout}

To validate the utility of YRNC10-gfp as a model for Yersinia ruckeri infection, it was important to verify that this modified strain had retained its ability to colonize and cause disease in exposed rainbow trout. Fish were immersion challenged with the YRNC10-gfp or the isogenic WT strain, and splenic bacterial loads were assessed as an indication of colonization and disease progression. Spleen colony counts were determined on Days 1, 3, 6, 9, 12, and 15 post-infection and a scatter plot of these data is presented in Fig. 1A. With the exception of Day 6, there were no significant differences in cfu $\mathrm{mg}^{-1}$ spleen tissue between WT- and YRNC10-gfp immersion exposed fish. Between 8 and $16 \mathrm{~d}$ after infection, a low level of mortality was ob-
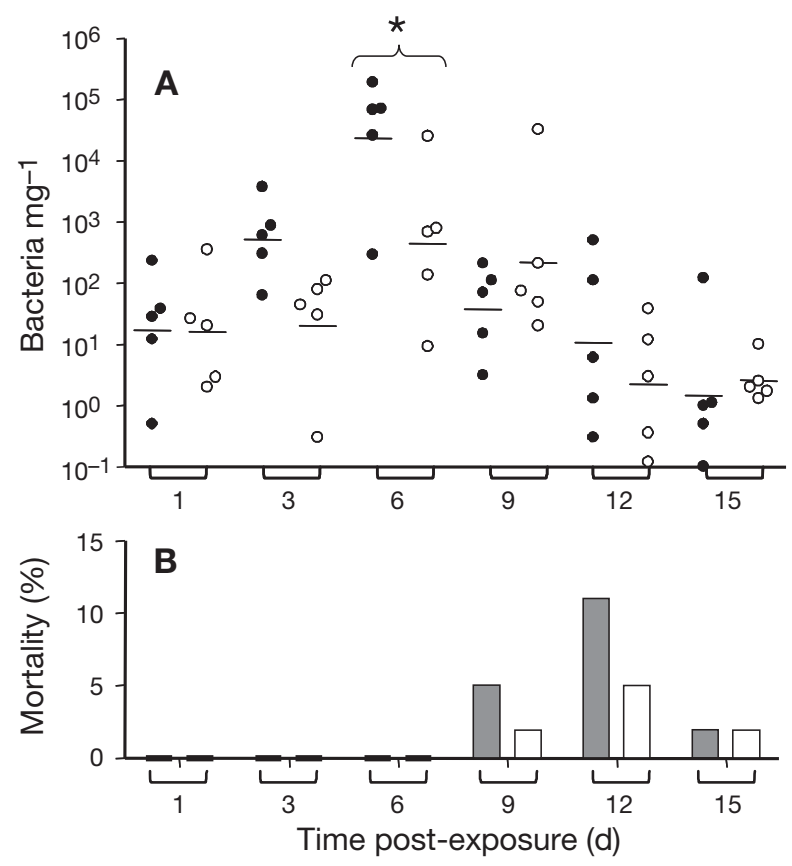

Fig. 1. Oncorhynchus mykiss. Splenic bacterial loads and mortality after immersion exposure to wild-type and gfpexpressing Yersinia ruckeri; 75 fish (50 to $100 \mathrm{~g}$ ) were immersion exposed to wild-type $Y$. ruckeri (०) or Y. ruckeri YRNC10-gfp ( $\bullet$ for $1 \mathrm{~h}$ at a concentration of $10^{8}$ bacterial $\mathrm{ml}^{-1}$. (A) Bacterial load in spleen of individual fish quantified at indicated times after exposure; *: significant difference $(p<0.05)$. Mean denoted by horizontal line. (B) Percent fish that died in intervals between sampling events. Shaded bars: wild-type Y. ruckeri; open bars: YRNC10-gfp

served in both WT- and YRNC10-gfp infected fish (Fig. 1B). Affected individuals showed gross pathology features typical of enteric redmouth disease, including hypertrophied spleen, exophthalmia, haemorrhaging in the lower intestine and frequently reddening around the lower jaw. The trends observed in bacterial splenic loads and mortality in the above experiment suggest that in vivo replication of the YRNC10-gfp strain is slower then that of its wild-type parent; as a result, this strain may be somewhat compromised in its ability to cause disease. To investigate whether there was a subtle difference in virulence, an immersion challenge was carried out using quadruplicate tanks per strain (Fig. 2). The total percent mortality observed for the YRNC10-gfp strain averaged $36 \%$ and ranged from 28 to $43 \%$. In comparison, the wild-type strain yielded an average of $65 \%$ mortality that ranged from 61 to $67 \%$. These data show a statistically significant reduction in mortality caused by the YRNC10-gfp strain and verify that this modified strain is impaired in its ability to cause disease compared to the wild type. Since YRNC10-gfp does not show any apparent growth defects on plate culture, the reduced virulence may be either due to GFP expression in vivo, or the GFP cas- 


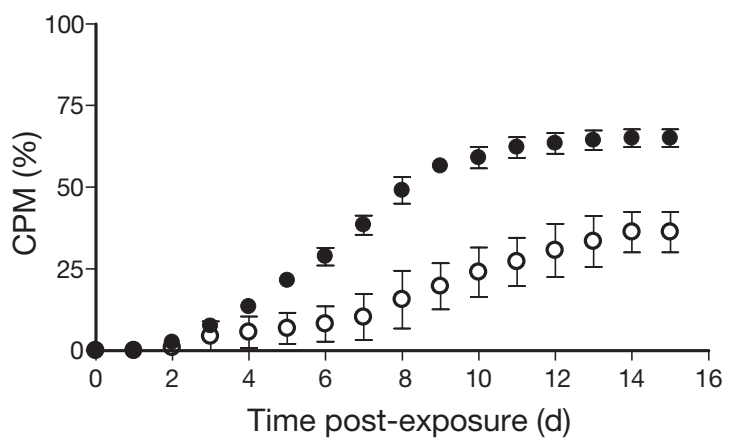

Fig. 2. Oncorhynchus mykiss. Mortality induced by immersion exposure to YRNC10-gfp or wild-type Yersinia ruckeri; 4 groups of 80 fish (average weight $5 \mathrm{~g}$ ) were immersion exposed to wild-type $Y$. ruckeri $(\bullet)$ or $Y$. ruckeri YRNC10-gfp (O) for $1 \mathrm{~h}$ at a concentration of $10^{8}$ bacterial ml ${ }^{-1}$. Cumulative percent mortality (CPM) was recorded daily for $15 \mathrm{~d}$ after exposure. Error bars represent standard deviation ( $\mathrm{n}=4$ replicates per strain)

sette insertion into a genomic region that contributes to virulence. Regardless, this finding does not invalidate the utility of YRNC10-gfp as a model for studying bacterial-host interactions, as the YRNC10-gfp strain readily colonizes trout, causes pathology indicative of ERM and induces significant mortality.

It should be noted that the media used for all plating assays, including those for the YRNC10-gfp strain, did not contain kanamycin and therefore were not selective for the $g f p-K m$ marker. In addition, all bacterial clones isolated from YRNC10-gfp-infected fish were found to be GFP fluorescent, indicating a high degree of stability of the $g f p-K m$ marker in this strain during infection. Mortality was also induced by intraperitoneal challenge with YRNC10-gfp (data not shown).

\section{Visualization of $g f p$-expressing Yersinia ruckeri in infected tissue and host cells by epifluorescence microscopy}

YRNC10-gfp cells can be distinguished from WT cells by plating (Fig. 3A) and are easily visualized as motile, non-cell associated bacteria within rainbow trout tissue wet-mounted slides (Fig. 3 and authors' unpubl. data). In addition to the freely motile bacteria, a small number of intracellular bacteria were observed
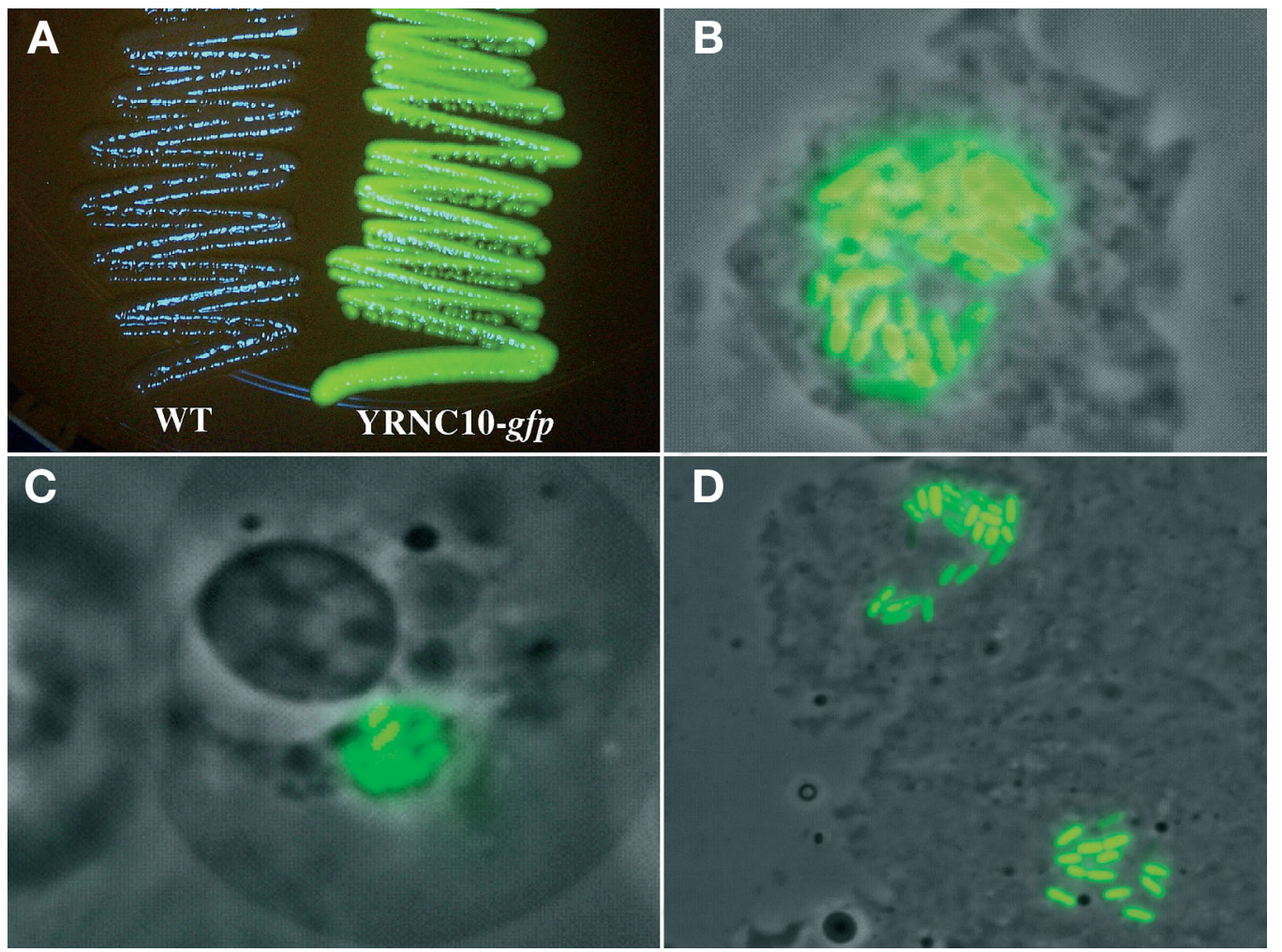

Fig. 3. Detection of green fluorescent protein (GFP)-producing Yersinia ruckeri in Oncorhynschus mykiss. Visualization of Y. ruckeri strain YRNC10-gfp. (A) Blue light-emitting diode (LED) illumination of cells grown on TSA. YRNC10-gfp cells were also observed in purified leukocytes enriched from (B) anterior kidney and (C) peripheral blood $48 \mathrm{~h}$ after intraperitoneal injection with approximately $10^{6}$ cells. (D) Homogenized anterior kidney tissue from a moribund fish from the immersion infection experiment in Fig. 1 (micrographs are merged phase contrast, epifluorescence images acquired with Nikon Eclipse E600 microscope at $55 \times$ magnification) 
in purified peripheral blood, anterior kidney and spleen leukocytes $48 \mathrm{~h}$ after intraperitoneal injection (Fig. 3B,C and authors' unpubl. data). Bacteria in these cells appeared to be contained within vacuole-like compartments. Formalin-killed YRNC10-gfp cells retained their GFP fluorescence, but were not detected 24 or $48 \mathrm{~h}$ after injection at a similar concentration. Large numbers of GFP-positive bacteria were also detected associated with infected spleen and kidney from several of the fish killed during the immersion challenge experiment described in the previous subsection (data not shown). The observation of GFP-positive YRNC10$g f p$ cells at several time points after infection and at all times when grown in culture suggests that the transcriptional fusion to the gfp gene in this strain resulted in constitutive production of GFP

\section{Detection of trout cells associated with $g f p$ - expressing Yersinia ruckeri by flow cytometry}

We examined whether flow cytometry could be used to measure the number, size and granularity of leukocytes that contained either intracellular or tightlyassociated extracellular $g f p$-expressing Yersinia ruckeri. Leukocytes isolated by gradient centrifugation from the peripheral blood, spleen and anterior kidney of sham-, Y. ruckeri WT- and YRNC10-gfp-injected fish were analyzed by flow cytometry. Dot plots of the cellular forward scatter (FSC) and side scatter (SSC) are shown in Fig. 4. Cells falling within the known lymphocyte, monocyte and neutrophil regions (Kollner et al. 2001, Stafford et al. 2001, Moritomo et al. 2003) were included in the R1 gate. Spleen and anterior kid-
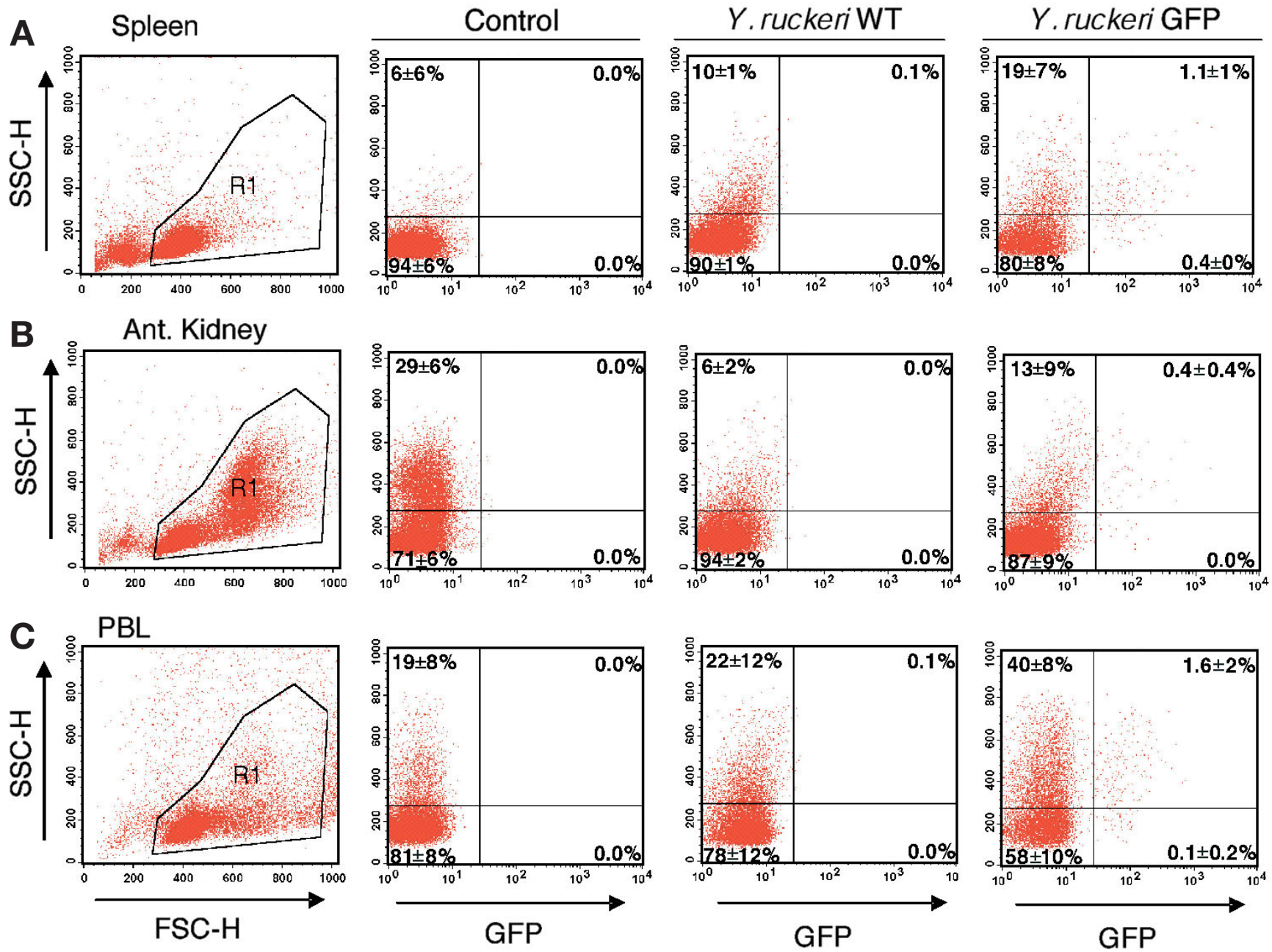

Fig. 4. Detection of green fluorescent protein (GFP)-producing Yersinia ruckeri in Oncorhynchus mykiss by flow cytometry. Leukocytes were isolated from the (A) spleen, (B) anterior kidney and (C) peripheral blood of (PBL) sham-, Y. ruckeri WT (wild-type)- and YRNC10-gfp (GFP+)-injected fish; size and granularity of cells determined by forward (FSC) and side scatter (SSC) plots ('control' = uninfected fish). Cells falling within the leukocyte R1 gate were analyzed for GFP fluorescence in the FL1 channel (abscissa) and for granularity (ordinate). Percentages in each quadrant represent average and standard deviation of 3 (sham-injected and Y. ruckeri WT-injected) or 4 ( Y. ruckeri GFP+)-injected fish 
ney tissues from both Y. ruckeri WT and YRNC10-gfp infections contained increased numbers of autofluorescent cells compared to sham-injected controls (data not shown). The nature of these cells is unknown and they were removed from the analyses by a second, R2 gate (data not shown). Small populations of trout cells were clearly identified in YRNC10-gfp-injected fish spleen, peripheral blood lymphocytes and anterior kidney samples which were not present in either the shaminjected or Y. ruckeri WT-injected fish (Fig. 4). The percentage of this population was highest in the peripheral blood $(1.6+2 \% ; \mathrm{n}=4)$ and lowest in the anterior kidney $(0.4+0.4 \% ; \mathrm{n}=4)$. The majority of the GFP-positive cells were in the non-lymphocyte quadrant (Fig. 4, compare percentage in upper right quadrant to lower right quadrant), consistent with known properties of rainbow trout phagocytic cells (Kollner et al. 2001, Moritomo et al. 2003). Interestingly, there was an increase in the percentage of larger, granular cells in the spleen and peripheral blood of infected fish compared to sham injected controls, while there was a decrease in these types of cells in the anterior kidney. These data are consistent with migration of leukocytes from the anterior kidney to the periphery during infection. In summary, a small percentage of rainbow trout cells could be identified by flow cytometry that either contained intracellular or tightly associated extracellular GFP-positive Y. ruckeri cells.

In this report we have described the construction and characterization of YRNC10-gfp, a virulent strain of Yersinia ruckeri that stably and constitutively expresses high levels of the green fluorescent protein. In addition, we used this strain to identify a small population of phagocytic cells containing intracellular bacteria. The small number of intracellular bacteria observed in these studies suggests that Y. ruckeri is predominantly an extracellular pathogen; however, a high proportion of bacteria could reside inside cells earlier in infection or in the chronic form of the disease. The detection of GFP-expressing fish pathogens by flow cytometry will allow the characterization of physiologically relevant trout cells that interact with pathogenic bacteria. This strain can also be easily identified in complex environmental samples containing multiple bacterial species (data not shown), making it especially

Editorial responsibility: Carey Cunningham, Aberdeen, UK useful for characterizing factors that allow its persistence and spread outside its host.

\section{LITERATURE CITED}

Busch (1978) Enteric red mouth disease (Haggerman strain). Mar Fish Rev 40:467-472

Chalfie M, Tu Y, Euskirchen G, Ward WW, Prasher DC (1994) Green fluorescent protein as a marker for gene expression. Science 263:802-805

de Lorenzo V, Herrero M, Jakubzik U, Timmis KN (1990) Mini-Tn5 transposon derivatives for insertion mutagenesis, promoter probing, and chromosomal insertion of cloned DNA in gram-negative eubacteria. J Bacteriol 172: 6568-6572

Kollner B, Blohm U, Kotterba G, Fischer U (2001) A monoclonal antibody recognising a surface marker on rainbow trout (Oncorhynchus mykiss) monocytes. Fish Shellfish Immunol 11:127-142

Ling SH, Wang XH, Xie L, Lim TM, Leung KY (2000) Use of green fluorescent protein (GFP) to study the invasion pathways of Edwardsiella tarda in in vivo and in vitro fish models. Microbiology (Reading) 146:7-19

Ling SH, Wang XH, Lim TM, Leung KY (2001) Green fluorescent protein-tagged Edwardsiella tarda reveals portal of entry in fish. FEMS Microbiol Lett 194:239-243

Moritomo T, Serata K, Teshirogi K, Aikawa H, Inoue Y, Itou T, Nakanishi T (2003) Flow cytometric analysis of the neutrophil respiratory burst of ayu, Plecoglossus altivelis: comparison with other fresh water fish. Fish Shellfish Immunol 15:29-38

O'Toole R, von Hofsten J, Rosqvist R, Olsson PE, Wolf-Watz H (2004) Visualisation of zebrafish infection by GFP-labelled Vibrio anguillarum. Microb Pathog 37:41-46

Southward CM, Surette MG (2002) The dynamic microbe: green fluorescent protein brings bacteria to light. Mol Microbiol 45:1191-1196

Stafford JL, McLauchlan PE, Secombes CJ, Ellis AE, Belosevic M (2001) Generation of primary monocyte-like cultures from rainbow trout head kidney leukocytes. Dev Comp Immunol 25:447-459

Stevenson RM (1997) Immunization with bacterial antigens: yersiniosis. Dev Biol Stand 90:117-124

Stretton S, Techkarnjanaruk S, McLennan AM, Goodman AE (1998) Use of green fluorescent protein to tag and investigate gene expression in marine bacteria. Appl Environ Microbiol 64:2554-2559

Valdivia RH, Falkow S (1996) Bacterial genetics by flow cytometry: rapid isolation of Salmonella typhimurium acid-inducible promoters by differential fluorescence induction. Mol Microbiol 22:367-378

Valdivia RH, Hromockyj AE, Monack D, Ramakrishnan L, Falkow S (1996) Applications for green fluorescent protein (GFP) in the study of host-pathogen interactions. Gene $173: 47-52$

Submitted: January 31, 2005; Accepted: July 12, 2005

Proofs received from author(s): November 21, 2005 